\title{
Take the Best or Look at the Rest? Factors Influencing "One-Reason" Decision Making
}

\author{
Ben R. Newell and David R. Shanks \\ University College London
}

\begin{abstract}
Aspects of an experimental environment were manipulated in 3 experiments to examine the parameters under which the "take-the-best" (TTB) heuristic (e.g., G. Gigerenzer \& D. G. Goldstein, 1996) operates. Results indicated TTB use to be more prevalent when the cost of information was high, when validities of the cues were known, and when a deterministic environment was used. However, large individual variability in strategy use was observed as well as a significant proportion of behavior inconsistent with TTB, expecially its stopping rule. The results demarcate some of the heuristic's boundary conditions and also question the validity of TTB as a psychologically plausible and pervasive model of behavior
\end{abstract}

When faced with the need to make a decision under conditions of uncertainty, there are typically several sources of information to which we can turn. Take, for example, predicting the winner of a horse race. We can gather information concerning the jockey, the trainer, the course, and the recent performance of the horse. We might then choose to integrate all this information, perhaps weighting some pieces, such as the identity of the trainer, as more important than others to reach a conclusion about the likelihood of the horse winning the race. An alternative to this integrative process is to single out a piece of information, such as the identity of the jockey, that we believe to be the best predictor of winning and base our decision solely on this information. Intuitively, basing a decision on a single piece of information seems to be an unwise policy. However, Gigerenzer and Goldstein (1996) have shown persuasively that such a simple "take-the-best" (TTB) heuristic can, under certain circumstances, prove to be as effective and sometimes better than integrating across a variety of sources of information. In this article, we explore the parameters within which this counterintuitive heuristic operates.

Gigerenzer (2000) claimed that we make decisions through the selection and application of a variety of fast and frugal heuristics contained in our cognitive "adaptive toolbox." The adaptive toolbox is a repertoire of rules or heuristics available to a species at a given point in its evolution (Gigerenzer \& Selten, 2001). One of the most basic algorithms in the adaptive toolbox, and the one that is the focus of this article, is the TTB strategy.

TTB is an extremely simple one-stop decision rule whose policy is "to take the best and ignore the rest" (Gigerenzer \& Goldstein, 1996). In contrast to the more classically rational decision strate-

Ben R. Newell and David R. Shanks, Department of Psychology, University College London, London, United Kingdom.

The support of the Economic and Social Research Council (ESRC) and The Leverhulme Trust is gratefully acknowledged. The work was part of the program of the ESRC Research Centre for Economic Learning and Social Evolution. We thank Mike Dougherty and Gerd Gigerenzer for their helpful comments on an earlier version of this article.

Correspondence concerning this article should be addressed to Ben R. Newell, Department of Psychology, University College London, Gower Street, London WC1E 6BT, United Kingdom. E-mail: b.newell@ucl.ac.uk gies in which information is integrated (e.g., linear regression, Bayesian reasoning), TTB simply uses the "best" piece of information applicable in a given situation (e.g., the identity of the jockey in our example). TTB operates according to two principles. The first - the recognition principle — states that in any given decision made under uncertainty, if only one among a range of alternatives is recognized, then the recognized alternative should be chosen (Goldstein \& Gigerenzer, 2002). That is, if you only recognize one jockey in the race, then choose the horse that he or she is riding. The second principle is invoked when more than one of the alternatives are recognized and the recognition principle cannot provide discriminatory information. In such cases, people are assumed to have access to a reference class of cues or features subjectively ranked according to their validities (where validity is defined as the probability that the feature will identify the correct alternative on a random selection of alternatives that differ on this feature). People are then thought to search the cues in descending order of feature validity until they discover a feature that discriminates one alternative from the others. Once this single discriminating feature has been found, it is used to make the decision. Thus, in our example, if the identity of the trainer is the most valid feature and one trainer has a better record than the others, then choose that trainer's horse.

TTB is, therefore, frugal because it bases the decision on a single feature and fast because it does not bother searching through and integrating information about other alternatives (i.e., it is noncompensatory). Thus, the TTB strategy can be thought of as comprising three basic building blocks: the search rule (search in order of validity), the stopping rule (stop after the first discriminating cue is discovered), and the decision rule (choose the outcome indicated by the first cue that discriminates). These elements of the strategy ensure that the demands placed on the cognitive system (e.g., working memory, processing capacity) are minimal and have led Gigerenzer and Todd (1999) to argue that TTB represents one of the purest forms of "bounded rationality," the term that Simon (1956) used to highlight the interconnectedness of the limitations of the mind and the structure of the environment in his model of human decision making. (For a full discussion of the fast, frugal, and rational nature of TTB, see Chater, Oaksford, Nakisa, \& Redington, in press.) 
Given the explicit and precise nature of TTB as described previously, it is straightforward to formulate and test hypotheses about its implementation (Bröder, 2000). Despite this apparent ease, there are few empirical tests of TTB, a situation that has not passed unnoticed (Bröder, 2000; Jones, Juslin, Olsson, \& Winman, 2000; Shanks \& Lagnado, 2000). It is true that a number of persuasive simulation studies have been carried out that show TTB to be a surprisingly accurate short-cut decision rule (e.g., Gigerenzer \& Goldstein, 1996), but such simulation data indicate nothing about TTB's adequacy as a description of actual human behavior.

Some evidence about the use of TTB-consistent strategies comes from a process-tracing experiment reported by Rieskamp and Hoffrage (1999). A task involving four alternatives (companies) and six cues (company variables, e.g., share capital, number of employees) was used, and participants were required to choose the company with the highest yearly profit. Participants' search patterns through the cues and alternatives were found to be consistent with noncompensatory decision strategies. For example, under high time pressure to make a decision compared with low time pressure, the time spent looking at each cue was more closely associated with the validities of the cues. Furthermore, participants adopted a "cuewise" search pattern (Payne, 1976), in which they tended to look at information about the same cue for different alternatives rather than amassing several pieces of information about the same alternative. This emphasis on examining the most valid cues is a hallmark of noncompensatory strategies and thus of TTB. However, as Rieskamp and Hoffrage (1999) readily pointed out, because of the overlap of these hallmark features with a number of strategies, such as Fishburn's (1974) lexicographic strategy and Tversky's (1972) elimination by aspects strategy, the process-tracing technique is limited in identifying a specific heuristic like TTB.

Jones et al. (2000), in a classification experiment, reported a more specific test of the TTB strategy. Limited support was found for TTB use, but given that all the cue information was presented simultaneously, it is debatable whether Jones et al.'s design constituted a fair test of TTB. Gigerenzer and Todd (1999) stressed the importance of the step-by-step nature of cue discovery in TTB. They argued that tasks in which the need for search is obviated are unsuitable for testing the boundedly rational TTB strategy because limited search through information is a central component of such heuristics (i.e., the search rule). Ensuring that cognitive, or better still economic, effort is necessary for the acquisition of information guarantees an appropriate environment for an empirical test of TTB.

Bröder (2000) used a design with the sequential presentation of information and combined it with a statistical inference technique to test the empirical validity of TTB. He tested both a strong hypothesis-that all participants in an experimental situation would adopt a TTB strategy — and a weak hypothesis - that only some participants would adopt TTB. Across four experiments, Bröder found no evidence for the strong hypothesis. In all Bröder's experimental conditions, a substantial number of participants exhibited behavior that violated the assumptions of TTB. In the final two experiments, participants took on the role of a stockbroker and were required to predict which of two shares from two fictional companies was the most profitable. To help them make their predictions, participants were able to buy information relating to the companies' financial status. This information took the form of binary cues (e.g., Is it an established company? Yes/No). On each trial participants were able to buy up to four pieces of information before making their prediction. This sequential buying of pieces of information, rather than the simultaneous and automatic provision of information, is the key element of the design that makes these experiments perhaps the best test of TTB to date.

Bröder (2000) recorded participants' search patterns from the share profitability task and used a multiple regression technique to analyze individual response vectors (for a detailed discussion of the statistical technique, see Bröder, 2002). Bröder classified 65\% of participants as conforming to a TTB strategy when the cost of information was high in both Experiments 3 and 4. In contrast, when the cost of information was lower or information was free, the number of TTB users dropped markedly to about $15 \%$.

The environment of Bröder's Experiments 3 and 4 was highly constrained to promote the use of TTB. First, participants were told the validities of the four cues before the start of the trials, allowing the subjective rank ordering of cues assumed by TTB to be established. Second, participants were only allowed to buy information in the order of the cue validities. Participants were forced to buy the best first, the second best second, and so on, thus presumably creating a bias to stop buying information upon reaching a discriminating cue. Despite these two elements of the environment strongly favoring TTB, 33\% of participants did not use TTB even when the cost of buying information was high.

Bröder's data are intriguing but they leave a number of questions unanswered. First, can participants learn the cue validities if they are not provided directly? Gigerenzer, Hoffrage, and Kleinbölting (1991) proposed that people construct the hierarchy of cue validities in their reference class through the operation of some frequency-encoding mechanism. This monitoring of the frequencies of co-occurrences in the environment appears plausible given the research into the effectiveness of frequency estimation (e.g., Hasher \& Zacks, 1984), but it remains to be tested empirically within this particular paradigm. Second, if participants can learn the correct validities, will they then buy information about each cue in the order of their validities as the search rule of TTB predicts? One can imagine a scenario in which a participant decides always to buy two or three pieces of information and is thus unconcerned about the order in which he or she obtains them, which would be a violation of TTB. Third, can we shed light on why a substantial proportion of participants fail to terminate their search (stop buying information) once they have discovered a discriminating cue, thereby violating the stopping rule of TTB?

Rather than relying on the statistical inference technique used by Bröder, we decided to investigate these questions using a processoriented approach (Payne, Bettman, \& Johnson, 1988; Rieskamp \& Hoffrage, 1999). As noted previously, some caveats are involved with such an approach; however, we reasoned that, by using an adapted form of Bröder's share prediction task, we could closely monitor the three key aspects of participants' behavior that are indicative of TTB use: (a) the amount of information bought on each trial, (b) the order in which information is bought, and (c) whether or not information is bought after information discriminating between the two options has been discovered. We argue that these three directly observable aspects of behavior provide perhaps a better, and certainly a complementary, insight into TTB use than that derived from Bröder's statistical inference procedure. 


\section{Experiment 1}

\section{Method}

Participants. Sixteen members of the University College London community took part in the experiment and were assigned equally to two groups: a high relative cost (HRC) group and a low relative cost (LRC) group. The 5 men and 11 women had a mean age of 22.7 years $(S D=2.9$; range $=19-28$ ).

Stimuli and design. The experiment used a share profitability prediction task similar to that used by Bröder (2000). Participants were presented with a series of two-alternative forced-choice decisions between the shares of two fictional companies (Share A and Share B). The shares were described by four binary cues with semantic labels concerning aspects of the company's financial status. The cues had validities of $.80, .75, .70$, and .69. The assignment of the validities to the four nominal cues was counterbalanced across participants, but the screen position of the cues was constant. For each share there were 16 distinct cue patterns and thus 120 possible paired comparisons. For each comparison, there was an associated probability of Share A being the most profitable. After each choice the posterior probabilities that the chosen share was most profitable were calculated according to Bayes's rule, assuming stochastic independence of the cues. A random number generator then determined which share became most profitable according to this probability. (See the Appendix for details of the patterns and probability calculations.) Participants were motivated by the incentive of winning UK $5 p$ or $10 p$ (depending on the group they were assigned to) for every correct prediction. (UK£1 = approximately U.S.\$1.54.)

In the training phase, cue information was supplied automatically and simultaneously to participants. At test, information was not provided automatically, but participants were able to buy information about each cue in any order. The cost of each piece of information was $1 \mathrm{p}$. Thus, the relative cost of information in the HRC condition was one fifth of the potential $5 \mathrm{p}$ profit for making a correct prediction, and in the LRC condition each piece cost one tenth of the potential 10p profit.

Procedure. At the start of the experiment, participants were given a brief verbal description of the experiment and were told they would be making decisions about the profitability of company shares. Participants were then given full written instructions on the screen, which they read through with the experimenter. Participants were told that on each trial they would be asked to choose a share (one of two alternatives) that they thought would turn out to be the most profitable. To help them make this choice, participants were provided with four pieces of information about the two companies that the shares were from: (a) Was the share trend positive over the last few months?, (b) does the company have financial reserves?, (c) does the company invest in new projects?, and (d) is it an established company? Participants were told that in making their predictions they should try to work out which pieces of information were most useful because not all the pieces were equally informative.

Participants then clicked a button to advance to the first trial. The screen layout for the training trials is shown in Figure 1. Sixty training trials were selected at random from the 120 possible paired comparisons of all 16-cue patterns. The trials were presented in an order randomly selected for each participant. Participants were required to make a choice by clicking on the "Choose Share A" or "Choose Share B" button. On clicking either button, the two windows below the choice buttons displayed the probability (expressed as a percentage) that the chosen share would be most profitable and the share computed by the program as most profitable on that trial. If the correct share was chosen, the private account window was incremented by $5 p$. On completion of the 60 training trials, a further set of instructions was displayed telling participants that for the next 120 trials, the information would no longer be automatically supplied to them but that instead they had to buy each piece. Participants were told that they could buy as many or as few pieces as they desired and in any order.
The screen for the test trials preserved the same basic layout as in Figure 1 ; however, to the right of the information windows four "Buy information" buttons were added (one for each piece of information). Clicking on these buttons changed the contents of the information window from ? to either Yes or No. Having bought as much or as little information as they wanted, participants made their choice. The most profitable share for that trial was then displayed in the window. If the correct share was chosen, the private account was incremented by $5 p$ in the HRC condition and $10 p$ in the LRC condition minus any money that had been "invested" to buy information. For example, a participant in the HRC condition who bought all four pieces of information and made a correct prediction had his or her account incremented by $1 \mathrm{p}$, that is, $5 \mathrm{p}-(4 \times 1 \mathrm{p})$. The probability (expressed as a percentage) of the chosen share being most profitable was not provided in the test phase. All 120 possible comparisons of the 16 cue patterns were presented in a different random order for each participant.

On completion of the 120 trials, participants were presented with a new screen and were asked to type in ratings (a number between 1 and 100) of the usefulness of each piece of information for helping them make their predictions. The discrimination rates for each cue (i.e., how often a cue had Yes for one company and No for the other) were the same (.5); thus, we assumed that participants would understand usefulness in terms of validity.

\section{Results and Discussion}

Earnings. Participants earned an average of $£ 4.17$ (SD $=$ $£ 0.52)$ in the HRC condition and $£ 6.94(S D=£ 1.77)$ in the LRC condition in addition to their fee for participation.

Proportion correct. The proportion of times that the share chosen was most profitable was $68.8 \%$ across the 60 training trials and $66.8 \%$ across the 120 test trials. Both proportions were well above the chance level of $50 \%$, training $t(15)=14.34$, test $t(15)=7.01$, both $p \mathrm{~s}<.0001$.

Estimated usefulness of information. The usefulness ratings of the four pieces of information were normalized by summing the ratings given to each cue and dividing each rating by this total for each participant. The mean normalized usefulness ratings for the four pieces of information are displayed in Table 1. We used the nonparametric Jonckheere test for ordered alternatives (Siegel \& Castellan, 1988) to analyze the ratings data. This test indicated a significant trend $(J=2.075, p<.05)$ across the four ratings in the predicted descending order from the objectively most useful to the objectively least useful piece of information, although the ratings for Cues 1 and 2 were slightly inverted. Even though we found a significant trend, planned comparisons between the usefulness ratings for pairs of cues (most valid vs. second most valid, second vs. third most valid, and third vs. least valid) failed to reveal any significant differences (all $p s>.2$ ).

Online ranking. During the test phase, participants were able to buy up to four pieces of information before making their share choice. The computer recorded the order in which information was bought and the cue value associated with the information. The computer recorded a value of 1 each time information about the most valid cue was bought, a value of 2 for the second most valid, a value of 3 for the third most valid, and a value of 4 for the least valid cue. These data provided an online measure of participants' ranking of the usefulness of each cue. A participant who has learned the usefulness of each piece of information perfectly should always buy information about the most valid cue first, then the second most valid, and so on, thus providing mean online rankings of $1,2,3$, and 4 , respectively. Table 1 displays the mean online rankings derived from this measure. A Jonckheere test for 


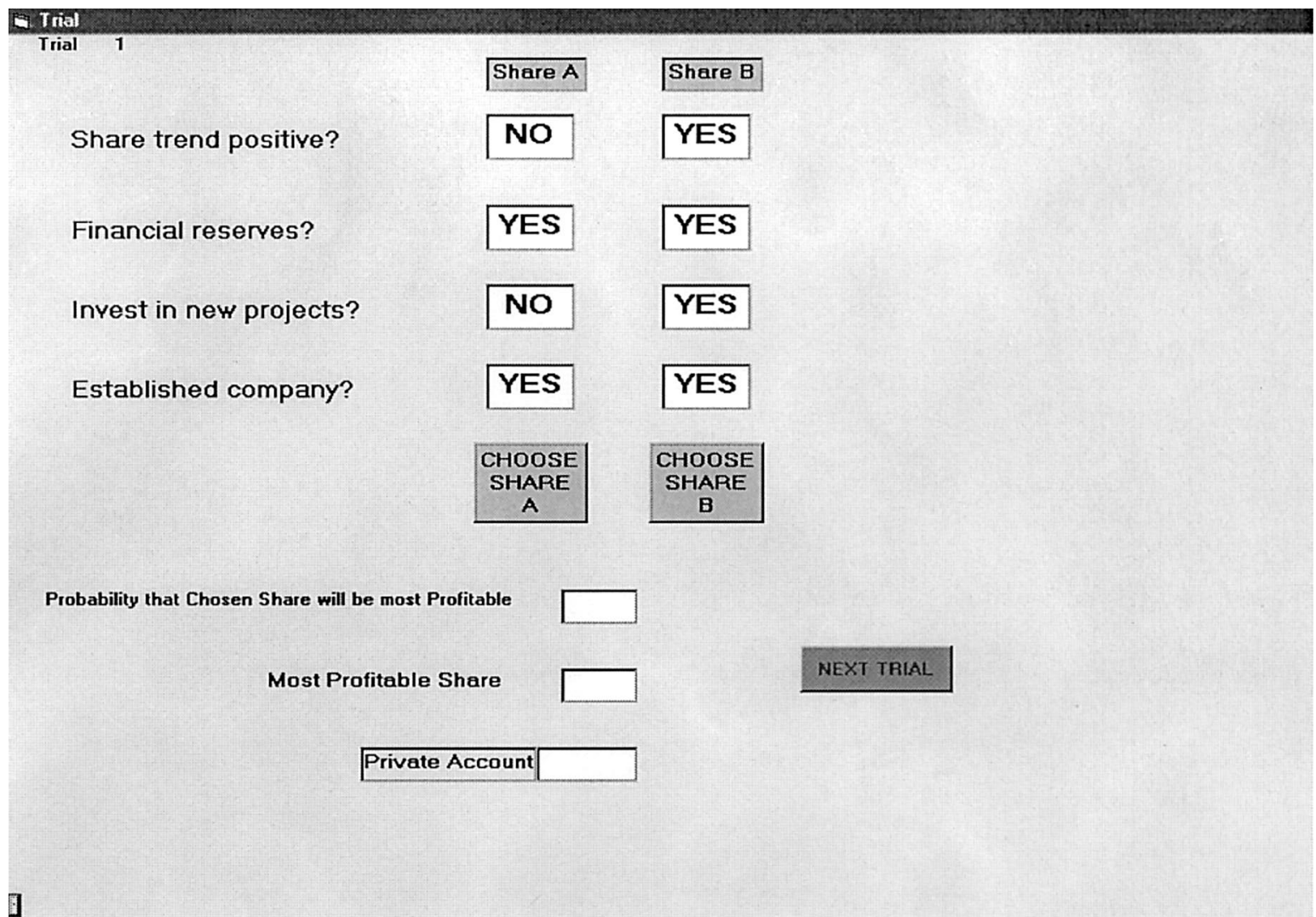

Figure 1. Screen layout during training trials of Experiment 1, Experiment 2, and the common labels condition of Experiment 3.

ordered alternatives indicated a significant trend $(J=2.053, p<$ $.05)$ across the four rankings in the predicted ascending order from the most valid to the least valid cue, although the rankings for the third and fourth most valid cues were quite markedly inverted. We acknowledge that this inversion may simply have been a reflection of the difficulty in learning the difference in validity between Cues 3 and 4 given that they differed only by .01 (.69 and .70, respectively). Again, although the trend was significant, planned comparisons revealed a significant difference only between the rankings for the second and third most valid cues, $t(15)=3.38$, $p<.01$.

Amount of information bought. The mean numbers of pieces of information bought on each trial in the HRC and the LRC conditions are shown in Table 2. A one-way analysis of variance (ANOVA) on the amount of information bought revealed a group difference, $F(1,15)=8.42, p<.05$, supporting the prediction that

Table 1

Group Data for Estimated Usefulness and Online Ranking of the Four Cues in Experiments 1-3

\begin{tabular}{|c|c|c|c|c|c|c|c|c|}
\hline \multirow{2}{*}{$\begin{array}{l}\text { Experiment and } \\
\text { Variable }\end{array}$} & \multicolumn{2}{|c|}{ Cue 1} & \multicolumn{2}{|c|}{ Cue 2} & \multicolumn{2}{|c|}{ Cue 3} & \multicolumn{2}{|c|}{ Cue 4} \\
\hline & $M$ & $S D$ & $M$ & $S D$ & $M$ & $S D$ & $M$ & $S D$ \\
\hline \multicolumn{9}{|l|}{ Experiment 1} \\
\hline Estimated usefulness & .28 & .10 & .29 & .11 & .22 & .09 & .21 & .09 \\
\hline Online ranking & 2.21 & 1.14 & 2.23 & .60 & 2.82 & .82 & 2.66 & .92 \\
\hline \multicolumn{9}{|l|}{ Experiment 2} \\
\hline Estimated usefulness & 41 & .07 & .28 & .10 & .21 & .10 & .10 & .07 \\
\hline Online ranking & 1.10 & .28 & 2.05 & .22 & 2.94 & .25 & 3.85 & .27 \\
\hline \multicolumn{9}{|l|}{ Experiment 3} \\
\hline Estimated usefulness & .36 & .07 & .30 & .05 & .20 & .04 & .13 & .07 \\
\hline Online ranking & 1.06 & .20 & 2.12 & .30 & 3.19 & .37 & 3.58 & .78 \\
\hline
\end{tabular}

Note. Estimated usefulness is the rating provided by participants at the end of the experiment (normalized across participants). Online ranking is derived from the order in which participants bought information in the test phase. Cue 1 is the most valid; Cue 4 is the least valid. 
participants in the LRC condition would buy more information per trial than those in the HRC condition.

In addition to the amount of information bought per trial, we examined the order in which participants bought information so that we could ascertain the proportion of trials on which participants continued to buy information after discovering a discriminating cue. In particular, we were interested in those trials on which a participant bought information about a discriminating cue (e.g., Yes for Share A and No for Share B) and then went on to buy information about another cue. Note that this measure does not take into account whether participants buy information in the order of their cue validities; it simply records those trials on which another piece of information was bought after a discriminating cue had already been found. The mean proportion of trials on which these unnecessary (as far as TTB is concerned) pieces of information were bought is shown in Table 2 .

A one-way ANOVA on the unnecessary information revealed a group difference, $F(1,15)=26.33, p<.001$, indicating that participants in the LRC condition bought unnecessary information on a greater proportion of trials than those in the HRC condition. The buying of unnecessary information on almost two thirds of trials indicates that participants in the LRC condition exhibited behavior clearly inconsistent with a TTB strategy. For those in the HRC condition, the amount of unnecessary information bought is much lower and plausibly within the limits of what might be expected from response errors or fluctuations in attention during the test phase. ${ }^{1}$

When information had a low cost relative to the profit available for a correct decision, only 1 participant (13\%) bought unnecessary information on less than $20 \%$ of trials. In contrast, when the relative cost was high 6 participants $(75 \%)$ exhibited this behavior. These figures compare favorably with the $15 \%$ and $65 \%$ of TTB users classified by Bröder (2000) in his low and high information cost conditions, respectively. Although this group level analysis is informative, an analysis of individual participant data revealed a more complex picture.

Individual participant data. The TTB algorithm assumes a subjective rank ordering of cues and a search through these cues in the order of their validities. At the group level, the data from Experiment 1 provide partial support for this assumption. The statistical analysis revealed significant trends in the predicted direction for both the estimation of information usefulness and the

Table 2

Group Data for Amount and Type of Information Bought at Test in Experiment 1

\begin{tabular}{lrrrrrr}
\hline & \multicolumn{2}{c}{$\begin{array}{c}\text { High relative } \\
\text { cost }\end{array}$} & & \multicolumn{2}{c}{$\begin{array}{c}\text { Low relative } \\
\text { cost }\end{array}$} \\
\cline { 2 - 3 } \cline { 6 - 7 } \multicolumn{1}{c}{ Variable } & $M$ & & $S D$ & & $M$ & $S D$ \\
\hline Amount of information & 1.36 & .63 & & 2.54 & .61 \\
Unnecessary information & .15 & .12 & & .64 & .24 \\
\hline
\end{tabular}

Note. Amount of information refers to the mean number of pieces of information bought on each trial. Unnecessary information refers to the proportion of trials on which information was bought after discovering a discriminating cue. This measure is conditionalized on whether any information was bought at all. online ranking. However, although these trends were significant, inspection of the means reveals only a small range in both the estimations and rankings and indeed an order reversal in both the estimation of usefulness of Cues 1 and 2 and in the online ranking for Cues 3 and 4. Individual participant data revealed that only Participant 9 provided estimations of usefulness in the order $1>$ $2>3>4$ and an online ranking in the order $1-2-3-4$. As Table 3 shows, this participant bought no information (i.e., guessed) on $68 \%$ of test trials. On the remaining $32 \%$ of trials on which he did buy information, he continued to buy information after discovering a discriminating cue on $28 \%$ of trials. Thus, although displaying very fast and frugal behavior, it was clearly inconsistent with TTB. Of the remaining 15 participants, 2 (Participants 6 and 5) provided the correct online ranking but estimated the order of information usefulness incorrectly. Table 3 shows that Participant 6 bought unnecessary information on $68 \%$ of trials and Participant 5 guessed on more than $80 \%$ of trials. Both participants, therefore, behaved in a manner inconsistent with a strict TTB strategy.

Only Participant 14, who never violated the stopping rule (bought no unnecessary information) and only violated the search rule for cues that were extremely close in validity (Cues 3 and 4), can be accurately classified as behaving in a manner wholly consistent with TTB.

In sum, analysis of individual data indicates that, even though participants in the HRC condition only bought unnecessary information on approximately $15 \%$ of trials, this value is an underestimate because of the contributions from participants who searched randomly through cues or simply guessed without buying any information on a large proportion of trials. It is also worth noting that even in the HRC condition 5 of 8 participants bought unnecessary information on more trials than the $5 \%$ baseline we computed (see footnote 1).

The results of Experiment 1 highlight the difficulties participants have in establishing and searching through a subjective rank order of cues. This could be taken as problematic for the TTB heuristic because the heuristic assumes the establishment of such rank orderings in situations involving a much larger number of cues with greatly varying validities. Alternatively, as we have suggested, it may just be that our experimental environment does not lend itself easily to the learning of cue validities. In his Experiments 1 and 2 (in which an extraterrestrial research cover story was used), Bröder (2000) provided participants with 50 training trials per cue to establish the correct cue hierarchy. Given that the dispersion in validities between our cues was only 11 (.80-.69) and that our participants had 60 trials to learn about four cues, it is quite possible that many participants found learning the validities too difficult.

In acknowledgment of this difficulty, in Experiment 2 we decided to help participants learn the usefulness of each piece of information by giving them a hint about the correct rank ordering. If we can be confident that participants search through cues in their order of usefulness, we will be able to obtain a more accurate assessment of the consistency of participants' behavior with the TTB heuristic, especially its stopping rule.

\footnotetext{
${ }^{1}$ In the General Discussion section we present an estimate of $.05(S D=$ .07 ) for the rate of such error responses.
} 
Table 3

Individual Participant Data for Experiment 1

\begin{tabular}{|c|c|c|c|c|c|}
\hline $\begin{array}{c}\text { Participant } \\
\text { no. }\end{array}$ & Condition & $\begin{array}{l}\text { Online } \\
\text { ranking }\end{array}$ & $\begin{array}{l}\text { Estimated } \\
\text { usefulness }\end{array}$ & $\begin{array}{l}\text { Unnecessary } \\
\text { information }\end{array}$ & $\begin{array}{c}\text { No } \\
\text { information } \\
\text { (guessing) }\end{array}$ \\
\hline 1 & HRC & $2-1-3-4$ & $2>1>3>4$ & .23 & .36 \\
\hline 3 & $\mathrm{HRC}$ & $2-3-4-1$ & $3>1>2>4$ & .05 & 0 \\
\hline 5 & HRC & $1-2-3-4$ & $1>3>2>4$ & .24 & .81 \\
\hline 7 & HRC & $4-1-3-2$ & $2>3=4>1$ & .08 & 0 \\
\hline 9 & HRC & $1-2-3-4$ & $1>2>3>4$ & .28 & .68 \\
\hline 12 & HRC & $3-2-4-1$ & $4>1=3>2$ & .04 & .08 \\
\hline 14 & HRC & $1-2-4-3$ & $1>2>4>3$ & 0 & 0 \\
\hline 15 & HRC & $3-2-1-4$ & $2>1>3>4$ & .30 & 0 \\
\hline 2 & LRC & $1-2-4-3$ & $1>3>4>2$ & .78 & .01 \\
\hline 4 & LRC & $4-2-3-1$ & $4>2>3>1$ & .83 & 0 \\
\hline 6 & LRC & $1-2-3-4$ & $1=2>4>3$ & .68 & 0 \\
\hline 8 & LRC & $2-3-4-1$ & $2>3>4>1$ & .75 & 0 \\
\hline 10 & LRC & $2-3-1-4$ & $1=3>2>4$ & .70 & 0 \\
\hline 11 & LRC & $3-2-4-1$ & $4>2>1>3$ & .56 & 0 \\
\hline 13 & LRC & $1-3-4-2$ & $1>3>4=2$ & .08 & 0 \\
\hline 16 & LRC & $3-2-1-4$ & $1=2=3=4$ & .76 & .82 \\
\hline
\end{tabular}

Note. Online ranking is derived from the order in which participants bought information in the test phase. Estimated usefulness is the rating provided by participants at the end of the experiment (normalized across participants). Unnecessary information refers to the proportion of trials on which information was bought after discovering a discriminating cue. This measure is conditionalized on whether any information was bought at all. For example, Participant 16 guessed on $82 \%$ of trials (99 of 120); on the remaining 18\% of trials (21 of 120), she bought unnecessary information on $76 \%$ (16 of 21). No information (guessing) refers to the proportion of trials on which participants did not purchase any information before making their decision. $\mathrm{HRC}=$ high relative cost LRC $=$ low relative cost.

\section{Experiment 2}

\section{Method}

Participants. Twelve members - 5 men and 7 women-of the University College London community took part in the experiment (mean age $=$ 27.5 years, $S D=5.7$; range $=21-39$ ).

Stimuli, design, and procedure. Experiment 2 was a replication of Experiment 1 with three exceptions. First, an LRC group was not included; for all participants information cost $1 \mathrm{p}$, and the payoff for a correct prediction was $5 \mathrm{p}$. Second, a hint regarding the rank order of usefulness of the four cues was provided on the computer monitor halfway through training (after 30 trials) and again at the start of the test phase. This hint indicated, for example, that knowing whether a company has a positive share trend was more useful than knowing whether it is an established company and so on. Usefulness was explained in terms of predictive utility. Third, participants were given a posttest questionnaire asking them about the strategy they had used in the test phase of the experiment.

\section{Results and Discussion}

Proportion correct. The proportion of times that the share chosen was the most profitable was $70.8 \%$ across the 60 training trials and $72.5 \%$ across the 120 test trials. These proportions were slightly higher than those obtained in Experiment 1, suggesting that the hint had a positive effect on participants' behavior. Consistent with Experiment 1, both proportions were well above the chance level of $50 \%$, training $t(11)=10.50$, test $t(11)=24.52$, $p s<.0001$.

Estimated usefulness of information and online ranking. Table 1 shows the mean estimated usefulness of each piece of information (normalized across participants) and the mean online ranking of the usefulness of each cue. Jonckheere tests for ordered alternatives indicated significant trends $(J=6.54$ and 7.82, respectively, $p \mathrm{~s}<.001)$ in the predicted order for both measures. The ranges between the four means for both measures are considerably larger than those observed in Experiment 1, suggesting that the hint about the usefulness of the information succeeded in encouraging participants both to buy information in the correct order and to estimate usefulness correctly. Further support for this interpretation comes from the planned comparisons, which revealed significant differences between the most valid and second most valid cues, second and third most valid cues, and third and least valid cues in all the comparisons for online ranking, $t \mathrm{~s}(11)=8.54,7.53$, and 9.87, respectively, $p \mathrm{~s}<.0001$, and in two of the three comparisons for estimated usefulness, $t \mathrm{~s}(11)=3.08,1.36$ (not significant), and 2.40, respectively, $p \mathrm{~s}<.05$.

Amount of information bought. The mean amount of information bought per trial was $2.39( \pm .68 S D)$ of a maximum of 4 and a minimum of 0 . This value is considerably higher than the amount bought in the HRC condition of Experiment 1 (1.36). One possibility for this increase is that providing participants with the hint about the usefulness of the information implied that all pieces of information had some value and were thus worth buying. In Experiment 1 some participants reported that they believed at least some of the pieces of information were of no help in making the correct predictions and so they never bought them. In extreme cases this led to some participants guessing on a large proportion of test trials. In Experiment 2 all participants bought at least one piece of information per trial on average.

The mean proportion of trials on which participants bought unnecessary information (i.e., continued to buy information after 
discovering a discriminating cue) was $.44( \pm .28 S D)$. This increase from the .15 observed in Experiment 1 is again perhaps due to the hint boosting participants' confidence in the relevance of all the pieces of information to the decision. The finding that participants bought unnecessary information on more than $40 \%$ of trials suggests that at a group level participants are displaying behavior inconsistent with a TTB strategy. However, in line with Experiment 1 , the group level analysis does not convey the spread of individual variability present in the data.

Individual participant data. Table 4 presents the individual participant data for Experiment 2. In contrast to Experiment 1, in which only 1 participant satisfied the assumption of rank ordering and search through cues in order of their usefulness, in Experiment 28 participants satisfied the assumption. Of these, 4 (Participants 4, 6, 8, and 10) bought unnecessary information on less than $20 \%$ of trials $($ mean $=12 \%$, range $=2-18 \%$ ) and 4 (Participants $2,9,11$, and 12 ) on more than $30 \%$ of trials (mean $=54 \%$, range $=31-82 \%$ ). We argue that the proportion of trials in this latter group is higher than could reasonably be attributed to response error or attention lapses (see footnote 1) and that these participants are, therefore, exhibiting behavior inconsistent with a TTB strategy, in particular with the strategy's stopping rule.

Of the remaining participants, 2 (Participants 1 and 2) produced the correct online ranking but estimated Cue 4 as more useful than Cue 3, and 2 (Participants 5 and 7) produced inaccurate rankings and estimations. All 4 of these participants bought unnecessary information on more than $50 \%$ of trials. Also shown in Table 4 is the reduction in the amount of guessing: It was almost completely eliminated. Presumably this is again a reflection of the usefulness hint implying that all pieces of information had some value and that, therefore, at least one piece was worth buying on each trial.

Posttest questionnaires. Participants were given a questionnaire in which they were asked to indicate the statement that best described their strategy or policy in the phase in which they had to buy information. Participants 4, 6, 7, 8, 9, and 10 classified their strategies as completely or predominantly consistent with a TTB strategy, and another 6 (Participants 1, 2, 3, 5, 11, and 12) classified their strategies as completely or predominantly inconsistent with a TTB strategy. In the latter case, participants explicitly stated that they always or predominantly bought more information after discovering a cue that discriminated between the two alternatives. Comparison of questionnaire responses with behavioral data demonstrated a high degree of coherence, with only 1 participant misclassifying her strategy as predominantly consistent with TTB (Participant 7) and another (Participant 9) misclassifying his strategy as completely consistent with TTB.

Both the behavioral and the questionnaire data point to a fairly clear division, with half of our participants behaving in a manner consistent with TTB and half behaving in a manner inconsistent. Why do some participants use a TTB strategy and some not? The participants who did use a strategy consistent with TTB earned an average of $£ 4.60$ compared with $£ 3.56$ earned by the nonusers. If financial gain was an important motivator in this environment (as the difference in amount of information bought between the LRC and HRC conditions in Experiment 1 seemed to indicate), all participants should use the TTB strategy. Anecdotal evidence from debriefing suggested that what some participants referred to as the "random element" of the experiment often led them to buy more information after discovering a discriminating cue. For example, they were frustrated by the fact that even if one share had Yes for all its pieces of information and the other had No, the latter share could still turn out occasionally to be most profitable. Thus, because the wrong share turned out to be most profitable on a few occasions, some participants were simply not confident enough in just one piece of discriminating information. Thus, we could say that these participants adopted a weight-of-evidence strategy whereby they bought extra information to increase their confidence in their choice.

Table 4

Individual Participant Data for Experiment 2

\begin{tabular}{ccccc}
\hline $\begin{array}{c}\text { Participant } \\
\text { no. }\end{array}$ & $\begin{array}{c}\text { Online } \\
\text { ranking }\end{array}$ & $\begin{array}{c}\text { Estimated } \\
\text { usefulness }\end{array}$ & $\begin{array}{c}\text { Unnecessary } \\
\text { information }\end{array}$ & $\begin{array}{c}\text { No } \\
\text { information } \\
\text { (guessing) }\end{array}$ \\
\hline 1 & $1-2-3-4$ & $1>2>4>3$ & .78 & 0 \\
2 & $1-2-3-4$ & $1>2>3>4$ & .53 & 0 \\
3 & $1-2-3-4$ & $1>2>4>3$ & .51 & 0 \\
4 & $1-2-3-4$ & $1>2>3>4$ & .02 & 0 \\
5 & $1-3-2-4$ & $1=3>2=4$ & .80 & 0 \\
6 & $1-2-3-4$ & $1>2>3>4$ & .61 & 0 \\
7 & $2-1-3-4$ & $2>1>3>4$ & .16 & 0 \\
8 & $1-2-3-4$ & $1>2>3>4$ & .31 & .0 \\
9 & $1-2-3-4$ & $1>2>3>4$ & .82 & 0 \\
10 & $1-2-3-4$ & $1>2>3>4$ & .52 & 0 \\
11 & $1-2-3-4$ & $1>2>3>4$ & & 0 \\
12 & $1-2-3-4$ & $1>2>3>4$ & & 0 \\
\hline
\end{tabular}

Note. Online ranking is derived from the order in which participants bought information in the test phase. Estimated usefulness is the rating provided by participants at the end of the experiment (normalized across participants). Unnecessary information refers to the proportion of trials on which information was bought after discovering a discriminating cue. This measure is conditionalized on whether any information was bought at all. No information (guessing) refers to the proportion of trials on which participants did not purchase any information before making their decision. 


\section{Experiment 3}

In Experiment 3 we decided to remove the random element from the experiment by creating a deterministic environment. The structure of the task remained the same as in Experiments 1 and 2, but the random number generator used to determine the most profitable share was removed from the program. On selecting Share A or Share B, the probability of that share being most profitable was still displayed to participants; however, instead of being referred to as a probability, it was termed the share value. If participants selected the share with the higher value, they made a correct prediction and their account was incremented by $5 \mathrm{p}$. In contrast to Experiments 1 and 2, every time participants selected the share with the higher value their account was incremented.

This deterministic environment allows us to make further predictions about the performance of a TTB strategy. In our stimulus set there were eight critical instances in which strict adherence to a TTB strategy led to an incorrect prediction. The most extreme example of these instances is the pair $1-0-0$ - 0 versus $0-1$ 1 - 1: Basing one's choice on the information provided by the first (and most valid) cue inevitably leads to an incorrect prediction because the remaining cues point in the opposite direction (i.e., they compensate for the first cue). Thus, if a participant's behavior is consistent with TTB, they should get all eight of these critical instances incorrect. Furthermore, their overall proportion correct should be in the region of the $93.3 \%$ (i.e., 120 - 8/120) predicted by a TTB strategy.

One disadvantage with the deterministic environment, however, is that it allows participants, to an even greater extent than in Experiments 1 and 2, to rely on a simple strategy of counting the number of Yes and No responses and then choosing the share with the greater number of Yes responses. Intuitively, it seems unlikely that people use such a simple summing strategy in real-world environments. For this reason, we included a further condition in Experiment 3 in which the cue labels were less transparent and, therefore, required participants to learn which value of each cue was indicative of a more profitable share. We suggest that distinct labels of this kind are potentially less susceptible, at least initially while the cue values are being learned, to a simple summing strategy.

\section{Method}

Participants. Twenty-four members -10 men and 14 women-of the University College London community took part in the experiment and were assigned to two groups of 12 each (mean age $=25.7$ years, $S D=6.0$, range $=16-39$ ).

Stimuli, design, and procedure. The difference of principal interest between the design of Experiment 3 and those of Experiments 1 and 2 was the removal of the stochastic element in the experimental environment. A random number routine was no longer used to generate the outcome on each trial; quite simply the share with the predetermined highest value was the winner, and every time a participant selected this share he or she earned $5 \mathrm{p}$. Two conditions were included: one in which the labels for the pieces of information were the same as those used in Experiments 1 and 2 (common labels, i.e., yes/no), and one in which a new set of semantic labels was used (distinct labels). This latter condition used the following distinct labels and answers: (a) Where does the company have the bulk of its operations? $(0=\mathrm{US}, 1=\mathrm{UK})$; (b) on which index are the company's shares listed? $(0=$ FTSE, $1=$ NASDAQ $)$; (c) is it an established company? $(0=$ yes, $1=$ no $)$; $(d)$ what is the employee turnover rate? $(0=$ high,
$1=$ low). Thus, the pattern $1-0-0-1$, for example, yielded the cues UK/FTSE/yes/low. The cue validities for the four pieces of information were the same as those used in Experiments 1 and 2. The distinct labels condition was included to provide a situation in which participants could not rely on a simple summing of Yes and No responses to arrive at a decision. Instead, participants were forced to learn the direction in which cues pointed before they were able to decide which share had the higher value. For example, they had to learn that a company with a low employee turnover was likely to have a higher share value than a company with a high employee turnover. Consistent with Experiment 2, participants in both conditions were provided with a hint after 30 training trials and again at the start of the test phase, indicating the order of usefulness of the four pieces of information.

A further modification in Experiment 3 was the extension of the posttest questionnaire to include the eight critical comparisons that are indicative of TTB use. Of the 120 comparisons in the test set, there are 8 in which use of a TTB strategy results in the wrong share selection. The questionnaire contained a sheet with all 8 critical comparisons displayed with their values (Yes/No or the appropriate label in the distinct labels condition) for each cue. We were interested in whether this simultaneous presentation of cue information in the paper format would affect participants' tendencies to use a TTB strategy. That is, even if participants used TTB while obtaining sequential information using the computer, would this behavior persist when all the necessary information was available simultaneously and at no cost?

\section{Results and Discussion}

Proportion correct. Table 5 shows the proportion of times that the share chosen was the share with the highest value. At test, it can be seen that neither group achieved the maximum proportion correct predicted by strict adherence to a TTB strategy (93.3\%). Test performance in both groups differed significantly from the maximum predicted by TTB but only marginally so in the common labels group, $t(11)=1.86, p=.09, t(11)=2.66, p<.01$, for the distinct labels group. All proportions correct were well above the chance level of $50 \%$ (all $p \mathrm{~s}<.05$ ). The difference in proportion correct between the common and distinct labels conditions was significant across the training trials, $t(11)=3.56, p<.01$, but not across the test trials, $t(11)=1.29, p>.1$. The difference in performance at training appears to have arisen from participants' initial difficulty in learning which value of a cue was best (e.g., whether NASDAQ or FTSE was indicative of share profitability) in the distinct labels condition. Mean proportion correct after the first 10 trials was only .59 in the distinct labels condition compared with .81 in the common labels condition. Our contention that the values of distinct labels would be more difficult to learn than the common labels and, therefore, less susceptible to a simple summing strategy is supported by the training trials data.

Estimated usefulness of information and online ranking. Table 1 shows the mean estimated usefulness of each piece of information (normalized across participants) and the mean online

Table 5

Proportion of Times Chosen Share Had Highest Value Across the 60 Training Trials and 120 Test Trials in Experiment 3

\begin{tabular}{lcc}
\hline \multicolumn{1}{c}{ Conditions } & Training & Test \\
\hline Common labels & .88 & .87 \\
Distinct labels & .74 & .82 \\
\hline
\end{tabular}


ranking of the usefulness of each cue. These means are collapsed across the two labels conditions because the measures did not differ. Jonckheere tests for ordered alternatives indicated a significant trend $(J=9.86$ and 9.19 , respectively, $p s<.001)$ in the predicted order for both measures. Consistent with Experiment 2, the ranges between the means for both measures indicate that the hint had the desired effect on participants' behavior. Further support for the effectiveness of the hint was indicated by the planned comparisons, which revealed significant differences between the most valid and second most valid cues, second and third most valid cues, and third and least valid cues in all the comparisons both for online ranking, $t \mathrm{~s}(23)=24.30,12.95$, and 1.83, respectively, $p \mathrm{~s}<$ .05 , one-tailed, and estimated usefulness, $t$ s $(23)=3.02,7.02$, and 3.96, respectively, $p \mathrm{~s}<.05$.

Amount of information bought. The mean amount of information bought per trial was $1.98(S D=.91)$ in the common label condition and $1.92(S D=.74)$ in the distinct label condition. The difference between these means was not significant, $t(11)=.13$, $p>$.1. The mean proportion of trials on which participants bought unnecessary information was .24 $(S D=.34)$ in the common labels condition and $.26(S D=.33)$ in the distinct labels condition. Again the difference between these means was not significant, $t(11)=$ $.14, p>.1$. Thus, at .25 , the mean proportion of trials on which unnecessary information was bought, collapsed across the two labels conditions, was higher than could reasonably be attributed to response error or attention lapses; therefore, at a group level behavior again appears to be inconsistent with adherence to a strict TTB strategy.

We suggested that the change to a deterministic environment in Experiment 3 would remove the random element of decision outcomes mentioned by some participants and thus lead to a reduction in both the mean amount of information bought per trial and the mean proportion of trials on which unnecessary information was bought. To test this suggestion, we performed a crossexperiment comparison between Experiment 2 and the common label condition of Experiment 3. A cross-experiment comparison is justified here because the experiments were run under the same conditions by the same experimenter using participants from the same pool. The only difference was that Experiment 3 used a deterministic environment and Experiment 2 a stochastic one. The mean proportion of trials on which unnecessary information was bought was considerably lower in Experiment 3 than in Experiment 2 (.26 vs. .44), and the difference was significant on a one-tailed test, $t(11)=1.79, p<.05$. In the case of amount of information bought per trial, even though the value was numerically lower in Experiment 3 (1.98 vs. 2.39), the difference was not significant, $t(11)=1.32, p>.1$, although it is likely that with more participants this difference would have reached significance. We found partial support, therefore, for our suggestion, but the change to a deterministic environment did not completely eliminate the tendency for some participants to adopt a weight-ofevidence strategy.

Individual participant data. In line with Experiments 1 and 2, analysis at the group level, although suggestive of nonadherence to a TTB strategy, does not serve to illustrate the high degree of individual variability across participants. Table 6 displays the individual data for the two label conditions.

Sixteen participants ( 6 from the distinct labels condition and 10 from the common labels condition) satisfied the assumption of rank ordering and search through cues in order of usefulness. Of these 16 participants, 11 (Participants 1, 5, 6, and 12 from the distinct labels condition and 2, 4, 5, 6, 7, 8, and 9 from the common labels condition) bought unnecessary information on less than $20 \%$ of trials $($ mean $=2 \%$, range $=0-18 \%$ ), and 4 (Participants 4 and 7 from the distinct labels condition and 10 and 11 from the common labels condition) bought unnecessary information on more than $40 \%$ of trials (mean $=65 \%$, range $=44-81 \%$ ). The remaining participant guessed on $86 \%$ of trials (Participant 3 , common labels).

Of the 8 participants who did not satisfy the assumption of ordering and search, 2 (Participants 2 and 11, distinct labels) provided incorrect rankings and estimations, 2 (Participants 3 and 8, distinct labels) inverted the ranking of Cues 3 and 4, 2 (Participants 1 from common labels and 9 from distinct labels) provided incorrect estimations, and 1 (Participant 10, distinct labels) guessed on $75 \%$ of trials and thus produced limited and incorrect ranking data. Finally, Participant 12 from the common labels condition provided the correct estimation of usefulness, but her online ranking data, rather than reflecting the validity of the cues, reflected the descending order of the "Buy information" buttons on the screen for her particular counterbalanced condition. It appears that having decided on a strategy to buy every piece of information on each trial (as supported by her posttest questionnaire) this participant simply bought the information in the most convenient manner given the screen layout.

Posttest questionnaires. Participants 2, 4, 5, 6, 7, 8, and 9 from the common labels condition and Participants 1, 2, 5, 6, 8, 9, 10, 11, and 12 from the distinct labels condition classified their behavior as predominantly or completely consistent with a TTB strategy. The remaining participants from both conditions classified their behavior as predominantly or completely inconsistent with a TTB strategy. Comparison of behavioral data with questionnaire responses revealed, in line with Experiment 2, a high degree of coherence: Only 3 participants misclassified their strategies: Participant 2 in the distinct labels condition reported using a TTB strategy when she was clearly not doing so, and Participant 3 in the common labels condition and Participant 10 in the distinct labels condition reported always buying at least one piece of information when, in reality, both participants guessed on more than $75 \%$ of trials.

Critical comparisons. TTB received some support from the critical comparison data. The use of TTB with Cue 1 as the most valid cue should yield incorrect selections on comparisons such as $0-1-1-1$ versus $1-0-0-0$ and $0-1-1-0$ versus $1-0-0-0$. The mean number of correct selections for these critical comparisons was 1.50 of 8 during the sequential trials on the computer and 3.96 of 8 when the comparisons were presented on paper. The lower number of correct selections in the computer trials indicates that, as predicted, TTB use was evident when information was presented sequentially and at a cost. Overall, in the computer trials 11 participants (46\%) made the incorrect selection on all eight critical comparisons. In contrast, only 2 participants $(8 \%)$ classified all eight comparisons incorrectly in the paper presentation. This large difference in TTB-consistent behavior between the simultaneous (paper) and sequential (screen) presentation of information highlights the importance of the step-by-step discovery of cue values for testing TTB (Gigerenzer \& Todd, 1999). Experiments in which information is presented simultaneously (e.g., Bröder, 2000, Ex- 
Table 6

Individual Participant Data for Experiment 3

\begin{tabular}{|c|c|c|c|c|c|}
\hline $\begin{array}{l}\text { Participant } \\
\text { No. }\end{array}$ & Condition & $\begin{array}{l}\text { Online } \\
\text { ranking }\end{array}$ & $\begin{array}{l}\text { Estimated } \\
\text { usefulness }\end{array}$ & $\begin{array}{l}\text { Unnecessary } \\
\text { information }\end{array}$ & $\begin{array}{c}\text { No } \\
\text { information } \\
\text { (guessing) }\end{array}$ \\
\hline 1 & CL & $1-2-3-4$ & $1>2=4>3$ & .71 & 0 \\
\hline 2 & CL & $1-2-3-4$ & $1>2>3>4$ & .01 & 0 \\
\hline 3 & $\mathrm{CL}$ & $1-2-3-4$ & $1>2>3>4$ & .12 & .86 \\
\hline 4 & CL & $1-2-3-4$ & $1>2>3>4$ & .06 & 0 \\
\hline 5 & $\mathrm{CL}$ & $1-2-3-4$ & $1>2>3>4$ & 0 & .05 \\
\hline 6 & CL & $1-2-3-4$ & $1>2>3>4$ & 0 & 0 \\
\hline 7 & CL & $1-2-3-4$ & $1>2>3>4$ & 0 & 0 \\
\hline 8 & $\mathrm{CL}$ & $1-2-3-4$ & $1>2>3>4$ & 0 & .02 \\
\hline 9 & CL & $1-2-3-4$ & $1>2>3>4$ & .02 & 0 \\
\hline 10 & CL & $1-2-3-4$ & $1>2>3>4$ & .66 & 0 \\
\hline 11 & $\mathrm{CL}$ & $1-2-3-4$ & $1>2>3>4$ & .44 & .01 \\
\hline 12 & $\mathrm{CL}$ & $2-3-4-1$ & $1>2>3>4$ & .93 & 0 \\
\hline 1 & DL & $1-2-3-4$ & $1>2>3>4$ & .18 & 0 \\
\hline 2 & DL & $1-2-3-4$ & $1>4>2>3$ & .83 & 0 \\
\hline 3 & DL & $1-2-3-4$ & $1>2>3>4$ & .32 & 0 \\
\hline 4 & DL & $1-2-3-4$ & $1>2>3>4$ & .72 & 0 \\
\hline 5 & DL & $1-2-3-4$ & $1>2>3>4$ & .01 & 0 \\
\hline 6 & DL & $1-2-3-4$ & $1>2>3>4$ & 0 & 0 \\
\hline 7 & DL & $1-2-3-4$ & $1>2>3>4$ & .82 & 0 \\
\hline 8 & DL & $1-2-4-3$ & $1>2>3>4$ & .22 & 0 \\
\hline 9 & DL & $1-2-3-4$ & $1=2=3=4$ & 0 & 0 \\
\hline 10 & DL & $1-4-2-3$ & $1>2>3>4$ & 0 & .75 \\
\hline 11 & DL & $1-2-4-3$ & $1>2>3>4$ & .01 & 0 \\
\hline 12 & DL & $1-2-3-4$ & $1>2>3>4$ & .04 & .27 \\
\hline
\end{tabular}

Note. Online ranking is derived from the order in which participants bought information in the test phase (see text for clarification). Estimated usefulness is the rating provided by participants at the end of the experiment (normalized across participants). Unnecessary information refers to the proportion of trials on which information was bought after discovering a discriminating cue. This measure is conditionalized on whether any information was bought at all. No information (guessing) refers to the proportion of trials on which participants did not purchase any information before making their decision. $\mathrm{CL}=$ common labels; $\mathrm{DL}=$ distinct labels.

periments 1 and 2; Jones et al., 2000) do not, therefore, provide a completely fair test of TTB.

Experiment 3 was designed to strongly favor the use of a TTB strategy. The random element of the stochastic environment used in Experiments 1 and 2 was replaced with a deterministic one; the hint explaining the usefulness of the pieces of information remained as did the training phase and motivating financial payoffs. These modifications appeared to promote the use of TTB. At an individual level, an impressive $75 \%$ of participants adhered to the TTB strategy, leaving only $25 \%$ of participants who preferred to err on the side of caution by seeking further weight of evidence.

It is important to emphasize that our results do not question the powerfulness of the TTB heuristic. Across the 120 test trials it was necessary to buy a minimum of 208 pieces of information (of a possible $480[120 \times 4])$ to uncover information that discriminated between the alternatives on every trial. Such a strategy has a $93.3 \%$ accuracy because of the eight misleading comparisons and, therefore, in a deterministic environment leads to a profit of $£ 3.50$ $([120 \times .93] \times 5 p-[208 \times 1 p])$. In contrast, buying one extra piece of information per trial (when there is another piece left to buy) leads to a profit of $£ 2.49([120 \times .93] \times 5 p-[309 \times 1 p])$. Of course, buying all four pieces of information on every trial results in a profit of only $£ 1.20([120 \times 5 \mathrm{p}]-[480 \times 1 \mathrm{p}])$. These latter two strategies both result in considerably lower profits than the $£ 3.00([120 \times .5] \times 5 p)$ that would be expected by simply guessing (buying no information) on every trial. Thus, in terms of financial gain, TTB is the best possible strategy, and it achieves this with more than $90 \%$ accuracy. However, even with this unambiguous financial advantage, not all participants adopted the strategy.

To illustrate this point, consider Participants 12 and 7 from the common labels condition. Participant 12 bought all four pieces of information on every trial and earned $£ 0.75$ at test. Participant 7 , who never bought unnecessary information (and never guessed), earned £3.47. This increase in profit of almost five times was achieved at the same level of accuracy across the test trials: $91.6 \%$.

\section{General Discussion}

The experiments presented here used a process-oriented approach to examine whether behavior in a two-alternative forcedchoice task with four information cues was consistent or inconsistent with a TTB strategy. Our key indicator of consistency with TTB was whether or not participants continued to search for (buy) information after discovering a cue that discriminated between the two alternatives. Such a weight-of-evidence strategy clearly violates the stopping rule of TTB.

Experiment 1 supported Bröder's (2000) finding that the relative cost of information affected the prevalence of TTB use: The higher the cost of information relative to the potential profit for making a 
correct choice, the higher is the number of TTB users. Experiment 1 also highlighted the difficulty participants had in learning the validities of the cues and thus the correct order in which to search through them. In much of the work on fast and frugal heuristics (Gigerenzer, 2000; Gigerenzer, Todd, \& the ABC Research Group, 1999), rather little attention is paid to how people learn validities and create the cue hierarchy in a particular reference class. Often the learning and decision/judgment aspects of tasks are amalgamated, and it is simply assumed that information relevant to the decision has been acquired through some unspecified or ill-specified experiential process. Experiment 1 demonstrated that only 1 participant $(6 \%)$ both estimated the validities of the cues correctly and searched through cues in the order of the validities. Even equipped with this knowledge, this participant chose to guess on more than $65 \%$ of test trials.

In Experiment 2 we attempted to promote the use of TTB's search rule further by telling participants the order of cue validities once during training and again before the test phase. This hint succeeded in leading the majority of participants to buy information in the correct order and estimate the usefulness of information correctly, but again $50 \%$ of participants continued to buy unnecessary information on more than $50 \%$ of the test trials on average.

Finally, in Experiment 3 we gave the TTB strategy the best possible chance by eliminating the probabilistic nature of the outcome of each choice. As predicted, this experiment had the highest proportion of TTB users-an impressive $75 \%$ - but we still found that $25 \%$ of those participants who satisfied the assumption of rank ordering and search violated the stopping rule on an average of $66 \%$ of test trials. In addition, fewer than half of the participants made the incorrect choice (an indicator of TTB adherence) for all eight critical comparison trials.

Our conclusions rest partly in determining the appropriate response error rate for our experimental environment. It is unrealistic to assume that TTB is a completely error-free heuristic; therefore, presumably unnecessary information will sometimes be bought by mistake. To estimate the frequency of such occasions, we calculated the proportion of trials on which unnecessary information was bought by those participants across all experiments whose online ranking, estimation, and self-report data were consistent with systematic use of TTB. These 15 participants bought unnecessary information on an average of $5 \%$ of trials $( \pm 7 \% S D)$. Thus, our absolute upper limit for response error was $19 \%$ (i.e., $5 \%$ plus $2 S D)$.

We also observed wide individual differences in all experiments, with some participants conforming very closely to the TTB strategy and others plainly violating it. Although the fast and frugal framework (Gigerenzer et al., 1999) allows for people to have access to numerous strategies or heuristics in their adaptive toolbox, it assumes that it is the environment that determines strategy selection and not the individual. Therefore, the individual differences we observed are not easily reconciled with the fast and frugal framework.

\section{Potential Limitations}

One potential criticism of our findings is our implicit assumption that when participants buy information this information is then incorporated into the decision-making process. As Rieskamp and Hoffrage (1999) pointed out, "Even if someone acquires a large amount of information, this does not necessarily mean that all of this information is used to make the decision" (p. 151). We agree that this criticism is valid but argue that, in the experimental environment used here, there is no reason (that we can think of) why participants would willingly sacrifice profit to buy information that they had no intention of using.

We are aware that dichotomizing participants into TTBconsistent and TTB-inconsistent groups is perhaps an oversimplification, and that it is quite possible that participants used a combination of both types of strategies (and others) in making their decisions. We also acknowledge that behavior can be consistent with one of the building blocks of TTB (e.g., the search rule) but inconsistent with another (e.g., the stopping rule). Therefore, classifying individuals as overall TTB or non-TTB users perhaps belies the complete picture. For example, Table 5 indicates that 10 of 12 participants in Experiment 2 exhibited behavior consistent with the search rule of TTB (as evidenced by the online rankings) but none satisfied the stopping rule (as evidenced by the purchase of unnecessary information). Similar patterns can be seen in Experiment 3 (e.g., Participants 9 and 11; see Table 6). We concede this point but stress that our aim in these experiments was to test TTB as a unitary heuristic (albeit one composed of three building blocks), and that observing behavior that is inconsistent with any aspect of the heuristic constitutes a violation of the explicit and precise patterns of behavior predicted by TTB.

We reiterate that our results do not challenge the power of the TTB heuristic. Those participants in Experiments 2 and 3 who exhibited TTB-consistent behavior earned an average of $£ 3.11$ across the 120 test trials compared with $£ 1.92$ for their non-TTBusing counterparts. This substantial increase in profit was achieved at a minimal loss in accuracy; in Experiments 2 and 3, TTB users achieved $81 \%$ correct and non-TTB users $83 \%$. We acknowledge that financial gain is not always perceived by participants as sufficient motivation for performing well (Friedman \& Massaro, 1998), but given the clear group difference between the relative cost conditions in Experiment 1, we argue that the payoff structure in the design we used was motivating to participants. If our assumptions about motivation are valid, then our results clearly show that some participants required a greater weight of evidence before making their choice than others.

\section{Weight-of-Evidence Strategy}

Is there any other evidence for the use of a weight-of-evidence strategy of the kind we observed in the three experiments? Harvey and Bolger (2001) identified "facilitating discrimination" as the key factor involved in motivating decision makers to improve their judgments and decisions. They proposed that "people's appreciation of the relative advantage of taking different options is subject to noise and that they collect information to increase their ability to discriminate between the attractiveness of options in the face of this uncertainty" (p. 296). Svenson's $(1992,1996)$ differentiation and consolidation theory also assumes a process in which alternatives are gradually differentiated from one another and people continue to acquire information until a particular candidate is "sufficiently superior for a decision" (Svenson, 1992, p. 143). Although these theories are typically applied to situations involving a greater number of alternatives and attributes (e.g., Harvey \& Bolger, 2001) than in the experiments presented here, it is clear 
that they support the conjecture that people are not always satisfied to make a decision on the basis of one piece of discriminating information, even when acquiring further information is potentially both financially and cognitively (in terms of effort) disadvantageous.

\section{Speed and Frugality}

TTB is said to be fast because it only searches for the amount of information necessary to discriminate between two alternatives. However, as Chater et al. (in press) noted, this assumption of speed is based on one particular view of cognitive architecture, namely that it is serial and that information in memory is searched at a constant rate. Such a serial architecture would give TTB an advantage over multiple regression for example, but presupposing seriality is perhaps premature. There is wide-ranging evidence for the viability of parallel architectures in which massive amounts of information can be searched simultaneously (e.g., Hopfield \& Tank, 1985; Rumelhart \& McClelland, 1986). Chater et al. (in press) also raised similar questions about the proposed advantage of the frugality of TTB. Several domains exist in which effective integration across multiple sources of information has been demonstrated experimentally and modeled successfully (e.g., face perception; Burton, Bruce, \& Hancock, 1999).

The claim that the classical rationality view of the mind is untenable because of the unrealistic amounts of computation required to perform, for example, Bayesian or regression calculation (Gigerenzer et al., 1999) can also be challenged. Shanks and Lagnado (2000) used the following example to demonstrate that a regression solution can be found in minimal time without any computation. Imagine a set of points, each represented by a peg on a two-dimensional board. Then attach a long, thin rod to the pegs using elastic bands at intervals along the rod. Minimizing the allocation of tension to the bands will then align the rod exactly according to the regression equation. Yet the elastic bands plainly do not compute the regression in any explicit sense.

Chater et al. (in press) mounted a similar line of argument against Gigerenzer et al.'s (1999) “cognitive limitations" claim. Chater et al. made the distinction between an algorithmic (cf. Marr, 1982) and rational (cf. Anderson, 1990) level of explanation of cognition. They noted that in a rational analysis calculating optimal behavior functions is extremely complex, and thus there does appear to be a contradiction between the intractability of these explanations and the time and memory limitations of the cognitive system. However, they argued that this contradiction ceases to be problematic when one realizes that people do not need to be able to calculate optimal behavior functions; they simply have to use successful algorithms. Consider, for example, a bird's ability to fly: A bird can fly perfectly well without knowing anything about aerodynamics or the computational intractability of aerodynamic calculations (Chater et al., in press).

Both these examples serve to illustrate that purportedly complex decision processes can be performed in simple ways, and that when a distinction is drawn between algorithmic and rational levels of explanation the perceived contradiction between the limitations of the cognitive system and the "demonic" proportions of required computations evaporates.

\section{Conclusion}

The fast and frugal heuristics program developed by Gigerenzer (2000) and colleagues (Gigerenzer \& Selten, 2001; Gigerenzer et al., 1999) is both important and influential. Our results highlight the powerfulness of one of the most fundamental heuristicsTTB-but also demonstrate that it is clearly not universally adopted by participants, even under conditions strongly constrained to promote its use. We acknowledge that Gigerenzer and his group do not assume that TTB is universal in the sense that it will be used by everyone regardless of the environment; nevertheless, our failure to find any circumstances, however restricted, in which the heuristic is almost universally used is, we believe, a real issue for TTB in particular and the fast and frugal approach in general (see also Newell, Weston, \& Shanks, in press).

Further experimental investigations of the kind reported here are necessary to ensure that the seductive appeal of "simple heuristics that make us smart" does not obscure the importance of empirical validation or distract us from our goal of understanding how, when, and why different decision heuristics and algorithms are successful.

\section{References}

Anderson, J. R. (1990). The adaptive character of thought. Hillsdale, NJ: Erlbaum.

Bröder, A. (2000). Assessing the empirical validity of the "take-the-best" heuristic as a model of human probabilistic inference. Journal of Experimental Psychology: Learning, Memory, and Cognition, 26, 13321346.

Bröder, A. (2002). Take the best, Dawes' rule, and compensatory decision strategies: A regression-based classification method. Quality \& Quantity, 36, 219-238.

Burton, A. M., Bruce, V., \& Hancock, P. J. B. (1999). From pixels to people: A model of familiar face recognition. Cognitive Science, 23, $1-31$.

Chater, N., Oaksford, M., Nakisa, R., \& Redington, M. (in press). Fast, frugal and rational: How rational norms explain behavior. Organizational Behavior and Human Decision Processes.

Fishburn, P. C. (1974). Lexicographic orders, utilities and decision rules. Management Science, 20, 1442-1471.

Friedman, D., \& Massaro, D. W. (1998). Understanding variability in binary and continuous choice. Psychonomic Bulletin \& Review, 5, 370389.

Gigerenzer, G. (2000). Adaptive thinking: Rationality in the real world. Oxford, England: Oxford University Press.

Gigerenzer, G., \& Goldstein, D. G. (1996). Reasoning the fast and frugal way: Models of bounded rationality. Psychological Review, 103, 650669.

Gigerenzer, G., Hoffrage, U., \& Kleinbölting, H. (1991). Probabilistic mental models: A Brunswikian theory of confidence. Psychological Review, 98, 506-528.

Gigerenzer, G., \& Selten, R. (2001). Rethinking rationality. In G. Gigerenzer \& R. Selten (Eds.), Bounded rationality: The adaptive toolbox (pp. 1-13). Cambridge, MA: MIT Press.

Gigerenzer, G., \& Todd, P. M. (1999). Fast and frugal heuristics: The adaptive toolbox. In G. Gigerenzer, P. M. Todd, \& the ABC Research Group (Eds.), Simple heuristics that make us smart (pp. 3-34). Oxford, England: Oxford University Press.

Gigerenzer, G., Todd, P. M., \& the ABC Research Group. (1999). Simple heuristics that make us smart. Oxford, England: Oxford University Press.

Goldstein, D. G., \& Gigerenzer, G. (2002). Models of ecological rationality: The recognition heuristic. Psychological Review, 109, 75-90. 
Harvey, N., \& Bolger, F. (2001). Collecting information: Optimizing outcomes, screening options, or facilitating discrimination. Quarterly Journal of Experimental Psychology: Human Experimental Psychology, 54(A), 269-301.

Hasher, L., \& Zacks, R. T. (1984). Automatic processing of fundamental information. American Psychologist, 39, 1327-1388.

Hopfield, J. J., \& Tank, D. W. (1985). "Neural" computation of decisions in optimization problems. Biological Cybernetics, 52, 141-152.

Jones, S., Juslin, P., Olsson, H., \& Winman, A. (2000, August). Algorithm, heuristic or exemplar: Process and representation in multiple cue judgment. In L. R. Gleitman \& A. K. Joshi (Eds.), Proceedings of the 22nd Annual Conference of the Cognitive Science Society (pp. 244-249). Mahwah, NJ: Erlbaum.

Marr, D. (1982). Vision: A computational investigation into the human representation and processing of visual information. San Francisco: Freeman.

Newell, B. R., Weston, N. J., \& Shanks, D. R. (in press). Empirical tests of a fast and frugal heuristic: Not everyone "takes the best." Organizational Behavior and Human Decision Processes.

Payne, J. W. (1976). Task complexity and contingent processing in decision making: An information search and protocol analysis. Organizational Behavior and Human Performance, 16, 366-387.

Payne, J. W., Bettman, J. R., \& Johnson, E. J. (1988). Adaptive strategy selection in decision making. Journal of Experimental Psychology: Learning, Memory, and Cognition, 14, 534-552.
Rieskamp, J., \& Hoffrage, U. (1999). When do people use simple heuristics and how can we tell? In G. Gigerenzer, P. M. Todd \& the ABC Research Group (Eds.), Simple heuristics that make us smart (pp. 141-167). Oxford, England: Oxford University Press.

Rumelhart, D. E., \& McClelland, J. L. (1986). On learning the past tenses of English verbs. In D. E. Rumelhart, J. L. McClelland, \& the PDP Research Group (Eds.), Parallel distributed processing: Explorations in the microstructure of cognition. Vol. 2: Psychological and biological models (pp. 216-271). Cambridge, MA: MIT Press.

Shanks, D. R., \& Lagnado, D. (2000). Sub-optimal reasons for rejecting optimality. Behavioral and Brain Sciences, 23, 761-762.

Siegel, S., \& Castellan, N. J. (1988). Nonparametric statistics for the behavioral sciences (2nd ed.). New York: McGraw-Hill.

Simon, H. A. (1956). Rational choice and the structure of environments. Psychological Review, 63, 129-138.

Svenson, O. (1992). Differentiation and consolidation theory of human decision making: A frame of reference for the study of pre- and postdecision processes. Acta Psychologica, 80, 143-168.

Svenson, O. (1996). Decision making and the search for fundamental psychological regularities: What can be learned from a process perspective? Organizational Behavior and Human Decision Processes, 65, 252-267.

Tversky, A. (1972). Elimination by aspects: A theory of choice. Psychological Review, 79, 281-299.

\section{Appendix}

Table A1

Probabilities That Share A or B Is More Profitable for the Different Cue Patterns, and the Likelihood Ratio (LR) Favoring A Over B

\begin{tabular}{lcccc}
\hline Cue & Pattern & $\mathrm{P}\left(\mathrm{A}=1 / \mathrm{C}_{i}\right)$ & $\mathrm{P}\left(\mathrm{B}=1 / \mathrm{C}_{i}\right)$ & LR (A:B) \\
\hline $\mathrm{C}_{1}$ & 10 & .80 & .20 & $4 / 1$ \\
& 01 & .20 & .80 & $1 / 4$ \\
$\mathrm{C}_{2}$ & 10 & .75 & .25 & $3 / 1$ \\
& 01 & .25 & .75 & $1 / 3$ \\
$\mathrm{C}_{3}$ & 10 & .70 & .30 & $7 / 3$ \\
& 01 & .30 & .70 & $3 / 7$ \\
$\mathrm{C}_{4}$ & 10 & .69 & .31 & $69 / 31$ \\
& 01 & .31 & .69 & $31 / 69$ \\
\hline
\end{tabular}

Note. $\mathrm{C}_{1}-\mathrm{C}_{4}$ refer to the individual cues (e.g., Established company?). A cue is diagnostic only when there is a YES for one share and a $N O$ for the other share. The Pattern column displays the two diagnostic patterns for each of the four cues $(1=Y E S, 0=N O) . \mathrm{P}\left(\mathrm{A}=1 / \mathrm{C}_{i}\right)$ refers to the probability that share $\mathrm{A}$ is more profitable $($ i.e., $\mathrm{A}=1)$ for a given cue pattern; $\mathrm{P}\left(\mathrm{B}=1 / \mathrm{C}_{i}\right)$ refers to the probability that share $\mathrm{B}$ is more profitable (i.e., $\left.\mathrm{B}=1\right)$ for a given cue pattern. To calculate the likelihood of a share from Company A being more profitable than one from Company B, the likelihood ratios corresponding to the particular patterns are multiplied. For example, if the pattern for Company A was 1111 and for Company B was 0000, then all four cues would be diagnostic, and the share from Company A would be $(4 / 1 \times 3 / 1 \times 7 / 3 \times 69 / 31)=62$ times more likely to be more profitable. In contrast, if the patterns were 0001 for Company $\mathrm{A}$ and 0000 for Company $\mathrm{B}$ only $\mathrm{C}_{4}$ would be diagnostic and the share from Company $\mathrm{A}$ would be $69 / 31$ or approximately twice as likely to be the more profitable.

Received October 26, 2001 